Sādhanā Vol. 39, Part 6, December 2014, pp. 1573-1583. (C) Indian Academy of Sciences

\title{
Dual solutions of Casson fluid flow over a stretching or shrinking sheet
}

\author{
PERI K KAMESWARAN ${ }^{1, *}$, S SHAW $^{2}$ and P SIBANDA ${ }^{2}$ \\ ${ }^{1}$ Department of Mathematics, National Institute of Science and Technology, \\ Berhampur 761 008, India \\ ${ }^{2}$ School of Mathematics, Statistics and Computer Science, University of \\ KwaZulu-Natal, Private Bag X01 Scottsville 3209, Pietermaritzburg, South Africa \\ e-mail: perikamesh@gmail.com
}

MS received 24 March 2014; revised 27 July 2014; accepted 11 August 2014

\begin{abstract}
The stagnation-point flow of an incompressible non-Newtonian Casson fluid over a stretching sheet in the presence of Soret and Dufour effects is investigated. The resulting partial differential equations are reduced to a set of nonlinear ordinary differential equations using similarity transformations and solved using the Matlab bvp4c package. A comparison is made with the results available in the literature and found to be in good agreement. Dual solutions for the velocity, temperature, concentration and skin friction were obtained for some special cases when the stretching parameter is negative. The effect of the Casson parameter on the skin friction, heat transfer and mass transfer rates is discussed.
\end{abstract}

Keywords. Casson fluid; stretching/shrinking sheet; Soret effect; Dufour effect.

\section{Introduction}

Boundary layer flow and heat transfer over a stretching sheet is significant due to its many applications in engineering processes such as in the extraction of polymer sheets, paper production, wire drawing and glass-fibre production. During the manufacturing process, a stretching sheet interacts with the ambient fluid both thermally and mechanically. The study of boundary layer flow caused by a stretching surface was initiated by Crane (1970) who gave an exact similarity solution in closed form. Mahapatra \& Gupta (2001) reconsidered the steady stagnation point flow towards a stretching sheet taking different stretching and stagnation point velocities and observed two different kinds of boundary layer structure near the sheet. Recently, several papers on the dynamics of the boundary layer flow over a stretching surface have appeared in the literature (Dutta et al 1985; Kameswaran et al 2012, 2013). Mukhopadhyay (2013) has studied the effects of Casson fluid flow and heat transfer over a nonlinearly stretching surface. She found

*For correspondence 
that temperature increases with an increase in nonlinear stretching parameter and the momentum boundary layer thickness decreases with an increase in Casson parameter.

When heat and mass transfer occur simultaneously in a moving fluid, the relationship between the fluxes and the driving potentials is complex. It has been observed that an energy flux can be generated not only by temperature gradients but also by concentration gradients. The energy flux caused by a concentration gradient is termed as the diffusion-thermo (Dufour) effect. On the other hand, mass fluxes can also be created by temperature gradients and this is termed as the thermal-diffusion (Soret) effect. Soret and Dufour effects have been utilized for isotope separation, in areas of hydrology, petrology, geosciences, etc. Srinivasacharya \& RamReddy (2011) have investigated the Soret and Dufour effects on heat and mass transfer along a semiinfinite vertical plate embedded in a non-Darcy porous medium. Soret and Dufour effects on the magnetohydrodynamic (MHD) flow of a Casson fluid over a stretched surface were also studied by Hayat et al (2012a, b) and Nawaz et al (2012). Chamkha \& Aly (2010) presented an analysis on heat and mass transfer in stagnation point flow of a polar fluid towards a stretching surface in porous media in the presence of Soret, Dufour and chemical reaction effects. Their study reveals that the velocity of fluid increased as the Soret number increased and the Dufour number decreased.

Casson fluids have a yield stress below which no flow occurs and a zero viscosity at an infinite rate of shear. The nonlinear Casson constitutive equation was derived by Casson (1959) and describes the properties of many polymers. At low shear rates when blood flows through small vessels, the blood flow is described by the Casson fluid model (McDonald 1974; Shaw et al 2009).

Non-Newtonian fluid flow generated by a stretching or shrinking sheet has many applications in industry. The flow of various non-Newtonian fluids over stretching or shrinking sheets was analysed by Liao (2003), Hayat et al (2008) and Ishak et al (2012). Stagnation point flow and heat transfer in a Casson fluid flow from a stretching sheet was studied by Mustafa et al (2012). An exact solution of the steady boundary layer flow of Casson fluid over a porous stretching or shrinking sheet was studied by Bhattacharyya et al $(2011,2013 \mathrm{a}, \mathrm{b})$. The effect of mass transfer on the magnetohydrodynamic flow of a Casson fluid flow over a porous stretching sheet was studied by Shehzad et al (2013). Hayat et al (2012a, b) studied the effects of mixed convection stagnation point flow of Casson fluid with convective boundary conditions. In their model, they showed that heat transfer characteristics depending on the embedded parameters.

The objective of this paper is to study the effect of the Casson parameter on the stagnation point flow of a Casson fluid over a permeable stretching or shrinking sheet subject to Soret and Dufour effects. We determine the dual fluid property solutions in the case when the surface is shrinking.

\section{Mathematical formulation}

Consider steady, incompressible two-dimensional flow of a Casson fluid near the stagnation point on a permeable stretching or shrinking sheet. The Cartesian coordinates $x$ and $y$ are along the surface and normal to it, $u$ and $v$ are the respective velocity components. The flow is generated by the stretching or shrinking of the sheet, caused by the simultaneous application of two equal forces along the $x$-axis. Keeping the origin fixed, the surface is stretched or shrunk with a linear velocity $u_{w}(x)=c x$, where $c$ is a constant with $c>0$ for a stretching sheet, $c<0$ for a shrinking sheet and $c=0$ for a static sheet. We assume that the rheological equation of state for 
an isotropic and incompressible flow of a Casson fluid can be written as (Nakamura \& Sawada 1988)

$$
\tau_{i j}=\left\{\begin{array}{ll}
\left(\mu_{B}+\tau_{y} / \sqrt{2 \pi}\right) 2 e_{i j} & \pi>\pi_{c} \\
\left(\mu_{B}+\tau_{y} / \sqrt{2 \pi_{c}}\right) 2 e_{i j} & \pi<\pi_{c}
\end{array},\right.
$$

where $\mu_{B}$ is plastic dynamics viscosity of the Casson fluid, $\tau_{y}$ is the yield stress of fluid, $\pi$ is the product of the component of deformation rate with itself, namely, $\pi=e_{i j} e_{i j}, e_{i j}$ is the $(i, j)$ th component of the deformation rate, and $\pi_{c}$ is the critical value $\pi$. The momentum, temperature and concentration equation under the above assumptions are written as

$$
\begin{gathered}
\frac{\partial u}{\partial x}+\frac{\partial v}{\partial y}=0 \\
u \frac{\partial u}{\partial x}+v \frac{\partial u}{\partial y}=u_{e} \frac{d u_{e}}{d x}+v\left(1+\frac{1}{\beta}\right) \frac{\partial^{2} u}{\partial y^{2}}+g \beta_{T}\left(T-T_{\infty}\right) \\
u \frac{\partial T}{\partial x}+v \frac{\partial T}{\partial y}=\alpha_{m} \frac{\partial^{2} T}{\partial y^{2}}+\frac{D_{m} K_{T}}{C_{s} C_{p}} \frac{\partial^{2} C}{\partial y^{2}} \\
u \frac{\partial C}{\partial x}+v \frac{\partial C}{\partial y}=D_{m} \frac{\partial^{2} C}{\partial y^{2}}+\frac{D_{m} K_{T}}{T_{m}} \frac{\partial^{2} T}{\partial y^{2}}
\end{gathered}
$$

The boundary conditions for equations (2)-(5) are given in the form:

$$
\begin{aligned}
& u=u_{w}(x)=c x, \quad v=0, \quad T=T_{w}(x)=T_{\infty}+b x, \quad C=C_{w}(x)=C_{\infty}+d x, \quad \text { at } \quad y=0, \\
& u \rightarrow u_{e}(x)=a x, \quad T \rightarrow T_{\infty}, \quad C \rightarrow C_{\infty}, \quad \text { as } \quad y \rightarrow \infty,
\end{aligned}
$$

where the ambient fluid moves with a velocity $u_{e}(x)=a x, a$ is a constant, $\beta=\mu_{B} \sqrt{2 \pi_{c}} / \tau_{y}$ is the Casson parameter, $g$ is the acceleration due to gravity, $\beta_{T}$ is the coefficient of thermal expansion, $T$ is the fluid temperature, $T_{\infty}$ is uniform ambient temperature, $\alpha_{m}$ is the effective thermal diffusivity, $D_{m}$ is the effective solutal diffusivity of the medium, $K_{T}$ is the thermal diffusion ratio, $C_{s}$ is the concentration susceptibility, $C_{p}$ is the specific heat capacity, $T_{m}$ is the mean fluid temperature. The continuity equation (2) is satisfied by introducing a stream function $\psi(x, y)$ such that

$$
u=\frac{\partial \psi}{\partial y} \quad \text { and } \quad v=-\frac{\partial \psi}{\partial x}
$$

where $\psi=(a v)^{\frac{1}{2}} x f(\eta), f(\eta)$ is the dimensionless stream function and $\eta=(a / v)^{\frac{1}{2}} y$. The velocity components are given by

$$
u=a x f^{\prime}(\eta) \text { and } \quad v=-(a v)^{\frac{1}{2}} f(\eta) .
$$

The temperature and concentration are represented as

$$
T=T_{\infty}+\Delta T \theta(\eta) \text { and } \quad C=C_{\infty}+\Delta C \phi(\eta)
$$


where $\theta(\eta)$ and $\phi(\eta)$ are the dimensionless temperature and dimensionless concentration. On using equations (7) and (8), equations (2)-(5) transform into the following two-point boundary value problem

$$
\begin{gathered}
\left(1+\frac{1}{\beta}\right) f^{\prime \prime \prime}(\eta)+f(\eta) f^{\prime \prime}(\eta)-f^{\prime 2}(\eta)+1+\lambda \theta(\eta)=0 \\
\frac{1}{P r} \theta^{\prime \prime}(\eta)+f(\eta) \theta^{\prime}(\eta)-f^{\prime}(\eta) \theta(\eta)+D_{f} \phi^{\prime \prime}(\eta)=0, \\
\frac{1}{S c} \phi^{\prime \prime}(\eta)+f(\eta) \phi^{\prime}(\eta)-f^{\prime}(\eta) \phi(\eta)+S r \theta^{\prime \prime}(\eta)=0, \\
f(0)=0, \quad f^{\prime}(0)=\frac{c}{a}, \quad f^{\prime}(\infty) \rightarrow 1, \\
\theta(0)=1, \quad \theta(\infty) \rightarrow 0, \\
\phi(0)=1, \quad \phi(\infty) \rightarrow 0,
\end{gathered}
$$

where the primes denote differentiation with respect to $\eta$. The non-dimensional constants in equations (9) - (14) are the buoyancy or free convection parameter $\lambda$, Prandtl number $\operatorname{Pr}$, Dufour parameter $D_{f}$, Schmidt number $S c$, Soret parameter $S r$ and the velocity ratio parameter $c / a$. These are defined as

$$
\begin{aligned}
\lambda & =\frac{g \beta_{T} b}{a^{2}}, \quad P r=\frac{v}{\alpha_{m}}, \quad D_{f}=\frac{D_{m} K_{T}\left(C_{w}-C_{\infty}\right)}{C_{s} C_{p} v\left(T_{w}-T_{\infty}\right)}, \quad S c=\frac{v}{D_{m}}, \\
S r & =\frac{D_{m} K_{T}\left(T_{w}-T_{\infty}\right)}{T_{m} v\left(C_{w}-C_{\infty}\right)} .
\end{aligned}
$$

\section{Skin friction, heat and mass transfer coefficients}

The quantities of practical interest in this study are the skin friction $C_{f}$. The shearing stress at the surface of the wall $\tau_{w}$ is given by

$$
\tau_{w}=\left(\mu_{B}+\frac{\tau_{y}}{\sqrt{2 \pi_{c}}}\right)\left[\frac{\partial u}{\partial y}\right]_{y=0}=\left(\mu_{B}+\frac{\tau_{y}}{\sqrt{2 \pi_{c}}}\right)\left(\frac{a^{3}}{v}\right)^{1 / 2} x f^{\prime \prime}(0) .
$$

The skin friction coefficient is defined as

$$
C_{f}=\frac{\tau_{w}}{\rho u_{e}(x)^{2}}
$$

and using equation (15) in the equation (16), we obtain

$$
C_{f} R e_{x}^{\frac{1}{2}}=\left(1+\frac{1}{\beta}\right) f^{\prime \prime}(0)
$$


The heat transfer rate at the surface flux at the wall is given by

$$
q_{w}=-k\left[\frac{\partial T}{\partial y}\right]_{y=0}=-k\left(T_{w}-T_{\infty}\right)\left(\frac{a}{v}\right)^{\frac{1}{2}} \theta^{\prime}(0) .
$$

The local Nusselt number is defined as

$$
N u_{x}=\frac{x q_{w}}{k\left(T_{w}-T_{\infty}\right)} .
$$

Using equation (18) in equation (19), the dimensionless Nusselt number can be represented as below

$$
\frac{N u_{x}}{R e_{x}^{\frac{1}{2}}}=-\theta^{\prime}(0)
$$

The mass flux at the surface of the wall is given by

$$
q_{m}=-D_{s}\left[\frac{\partial C}{\partial y}\right]_{y=0}=-D_{s}\left(C_{w}-C_{\infty}\right)\left(\frac{a}{v}\right)^{\frac{1}{2}} \phi^{\prime}(0) .
$$

The local Sherwood is defined as

$$
S h_{x}=\frac{x q_{m}}{D_{s}\left(C_{w}-C_{\infty}\right)} .
$$

Using (21) in (22) the dimensionless Sherwood number obtained as

$$
\frac{S h_{x}}{\operatorname{Re}_{x}^{\frac{1}{2}}}=-\phi^{\prime}(0)
$$

where $R e_{x}=x u_{e}(x) / v$ is the local Reynolds number.

\section{Results and discussion}

\begin{tabular}{|c|c|c|c|c|c|c|}
\hline \multirow[b]{2}{*}{$c / a$} & \multicolumn{2}{|c|}{ Present study } & \multicolumn{2}{|c|}{ Bhattacharyya (2011) } & \multicolumn{2}{|c|}{ Ishak et al (2010) } \\
\hline & 1st solution & 2nd solution & 1st solution & 2nd solution & 1st solution & 2nd solution \\
\hline-0.25 & 1.4022408 & & 1.4022405 & & 1.402241 & \\
\hline-0.50 & 1.4956698 & & 1.4956697 & & 1.495670 & \\
\hline-0.75 & 1.4892982 & & 1.4892981 & & 1.489298 & \\
\hline-1.00 & 1.3288169 & 0 & 1.3288169 & 0 & 1.328817 & 0 \\
\hline-1.15 & 1.0822312 & 0.1167021 & 1.0822316 & 0.1167023 & 1.082231 & 0.116702 \\
\hline-1.20 & 0.9324734 & 0.2336497 & 0.9324728 & 0.2336491 & 0.932474 & 0.233650 \\
\hline-1.2465 & 0.5842817 & 0.5542962 & 0.5842915 & 0.5542856 & 0.584295 & 0.554283 \\
\hline
\end{tabular}

The system of ordinary differential equations (9)-(11) subject to the boundary conditions (12)(14) were solved using the Matlab bvp4c ODE solver. To benchmark the numerical results, we have compared our results for the skin friction coefficient with those reported by Bhattacharyya

Table 1. Comparison of $f^{\prime \prime}(0)$ values with Bhattacharyya (2011) and Ishak et al (2010) for $\lambda=\operatorname{Pr}=$ $D f=S c=S r=0$ and $\beta=10^{8}$. 
Table 2. Critical $c / a$ value for different Casson parameter $\beta$.

\begin{tabular}{cccc}
\hline & $\beta=3.5$ & $\beta=4.0$ & $\beta=4.5$ \\
\hline$(c / a)_{\text {crit }}$ & -1.25182704 & -1.25182390 & -1.25181905 \\
\hline
\end{tabular}

(2011) and Ishak et al (2010) in table 1. The results are in very good agreement thereby lending confidence as to the accuracy of the present method.

This investigation confirms that the existence and uniqueness of solution depends on the stretching ratio parameter. We found that for a stretching sheet, solutions of equations (9)(11) may be found for all values of $c / a$, while for a shrinking surface, solutions exist only for $c / a>(c / a)_{\text {crit }}$, where $(c / a)_{c r i t}$ is a critical value of $c / a$ which depends on other parameters. There are no solutions when $c / a<(c / a)_{c r i t}$. Dual solutions of the fluid properties appear when $(c / a)_{\text {crit }}<c / a<-1$. The critical value $(c / a)_{\text {crit }}$ for different Casson parameters is given in table 2. It is evident that the critical value of $c / a$ is not significantly influenced by the Casson parameter $\beta$ within the chosen range.

In the absence of Soret and Dufour effects, for a particular values of other parameters, in order to validate our results, we have compared the results of heat transfer $-\theta^{\prime}(0)$ for various values of $c / a$ (table 3.) with those of Wang (2008) and found that the results are excellent in agreement. It can also be noticed that for the values of $c / a<0$ heat transfer decreases and increases for $c / a>0$.

Table 4 shows that the values of heat transfer coefficient for different $c / a$ and $\beta$. It is observed that heat transfer rate increases with an increase in ratio of $c / a$.

The dual solution is of the skin friction, heat transfer and mass transfer coefficients for different values of the Casson parameter are shown in figure 1. The Casson parameter $\beta$ is inversely proportional to the yield stress, hence a decrease in the Casson parameter indicates an increase in the yield stress. With an increase in $\beta$, the skin friction coefficient increases in the case of the first solution but is reduced in the second solution. It is interesting to note that the values of the heat transfer rate (Nusselt number) and mass transfer rate (Sherwood number) coincide when plotted against the stretching ratio parameter $c / a$ as shown in figure 1(b). An increase in the Casson parameter enhances both the heat and mass transfer rates. This is because the thermal and concentration boundary layer thicknesses decrease with $\beta$ causing the temperature and concentration gradients at the surface to increase.

Two velocity and temperature solutions were obtained when $c / a<-1$. These solutions are shown in figure 2 for three different values of $c / a=-1.23,-1.24,-1.25$. As observed by Merkin (1985), Merrill et al (2006), Paullet \& Weidman (2007), Postelnicu \& Pop (2011), the

Table 3. Comparison of $-\theta^{\prime}(0)$ with Wang for particular value of $c / a$.

\begin{tabular}{lcc}
\hline$c / a$ & Present study & Wang (2008) \\
\hline-0.25 & 0.6685728 & 0.66857 \\
-0.5 & 0.5014476 & 0.50145 \\
-0.75 & 0.2937625 & 0.29376 \\
0 & 0.8113013 & 0.811301 \\
0.1 & 0.8634517 & 0.86345 \\
0.2 & 0.9133028 & 0.91330 \\
0.5 & 1.0514584 & 1.05239 \\
1 & 1.2533141 & 1.25331 \\
\hline
\end{tabular}


Table 4. Heat transfer coefficient $-\theta^{\prime}(0)$ for different values of $c / a$ and $\beta$ for fixed values of $\lambda=0.01=$ $P r=D f=S c=S r=0.1$.

\begin{tabular}{lcccccccc}
\hline & \multicolumn{2}{c}{$\beta=3.5$} & & \multicolumn{2}{c}{$\beta=4.0$} & & \multicolumn{2}{c}{$\beta=5$} \\
\cline { 2 - 3 } \cline { 7 - 8 }$c / a$ & 1st solution & 2nd solution & & 1st solution & 2nd solution & & 1st solution & 2nd solution \\
\hline-1.25 & 0.0291102 & 0.0583329 & & 0.0324337 & 0.0613653 & & 0.0371985 & 0.0657208 \\
-1.20 & 0.053657 & 0.1128705 & & 0.0494951 & 0.1154347 & & 0.0435444 & 0.1191202 \\
-1.10 & 0.2132429 & 0.1549802 & & 0.2067033 & 0.1571813 & & 0.1974351 & 0.1603466 \\
-1.00 & & 0.1822328 & & 0.1841972 & & 0.1870228 \\
\hline
\end{tabular}

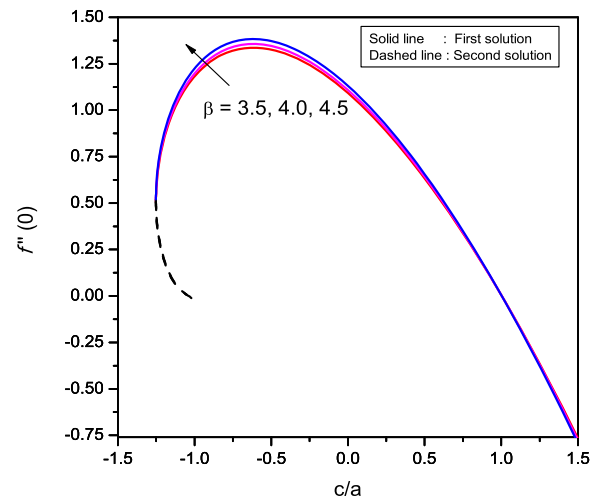

(a)

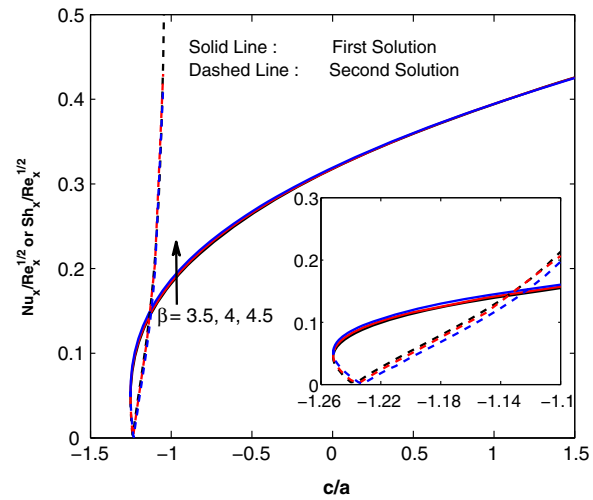

(b)

Figure 1. Effects of the stretching rate $c / a$ on the (a) skin friction and (b) heat transfer/mass transfer rates when $\lambda=0.01, \operatorname{Pr}=D_{f}=S c=S r=0.1$.

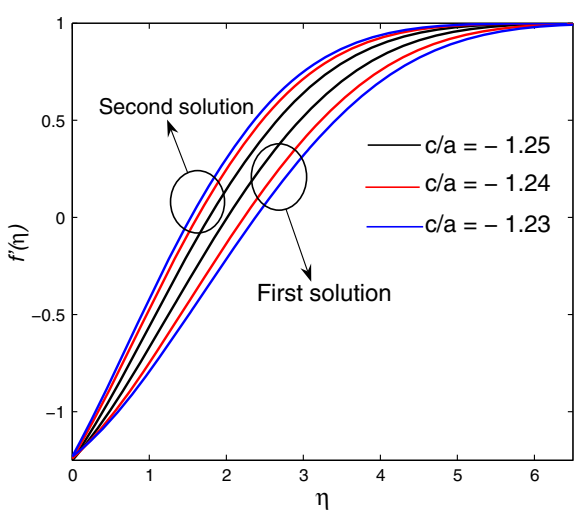

(a)

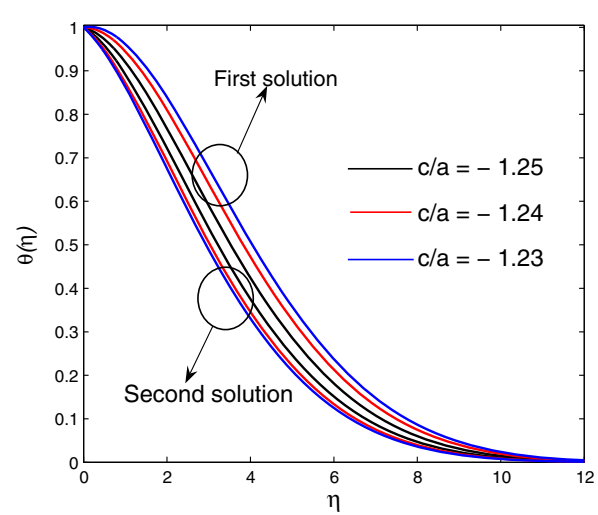

(b)

Figure 2. Effects of the stretching rate $c / a$ on the (a) velocity and (b) temperature solutions when $\beta=$ 3.1, $\lambda=0.01, \operatorname{Pr}=D_{f}=S c=S r=0.1$. 


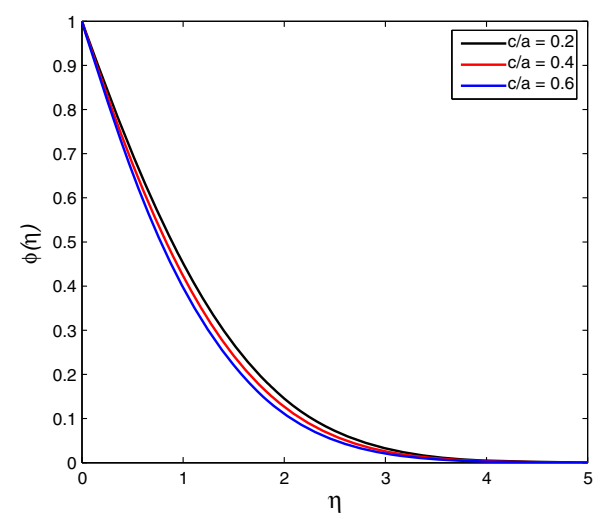

(a)

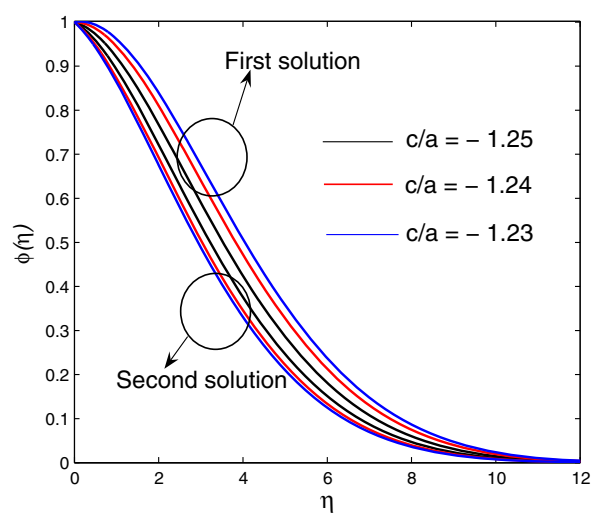

(b)

Figure 3. Effects of the stretching rate $c / a$ on (a) the concentration when $\beta=0.5, \lambda=1, \operatorname{Pr}=$ $0.7, D_{f}=0.5, S c=S r=0.8$ and (b) dual concentration solutions when $\beta=3.1, \lambda=0.01, \operatorname{Pr}=D_{f}=$ $S c=S r=0.1$.

first solution is stable and physically realizable, while the second solution is unstable. Although the second solution seems to be deprived of physical significance, it is interesting in the theory of nonlinear differential equations since a similar equation may appear in other situations where the solution could have physical significance. As the shrinking ratio increases, the free stream velocity decreases reducing both the momentum and thermal boundary layer thicknesses.

The effect of the stretching $(c / a>0)$ and shrinking $(c / a<0)$ ratios on the concentration profiles are shown in figure 3 . With an increase in the stretching ratio, the velocity of the fluid increases which in turn reduces the concentration layer thickness. In the case of a shrinking sheet we obtain dual solutions. For the first solution, the concentration boundary layer thickness increases with increase $c / a$ while for second solution in figure 3(b), the concentration profiles decrease with the shrinking ratio.

The influence of the Casson parameter on the velocity, temperature and concentration profiles is shown in figures 4 and 5 when $c / a=-1.25$. The magnitude of the velocity is greater in the

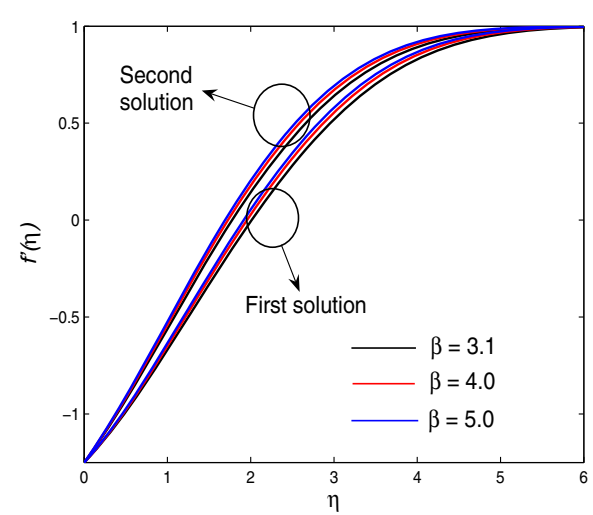

(a)

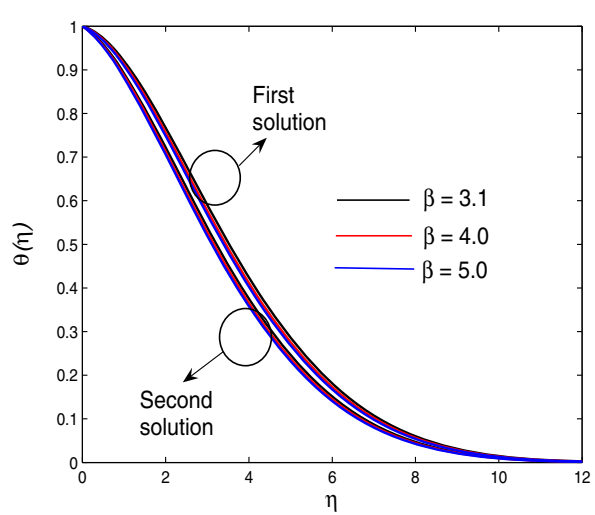

(b)

Figure 4. Effects of the Casson parameter on the (a) velocity and (b) temperature profiles when $\lambda=$ $0.01, \operatorname{Pr}=D_{f}=S c=S r=0.1, c / a=-1.25$. 


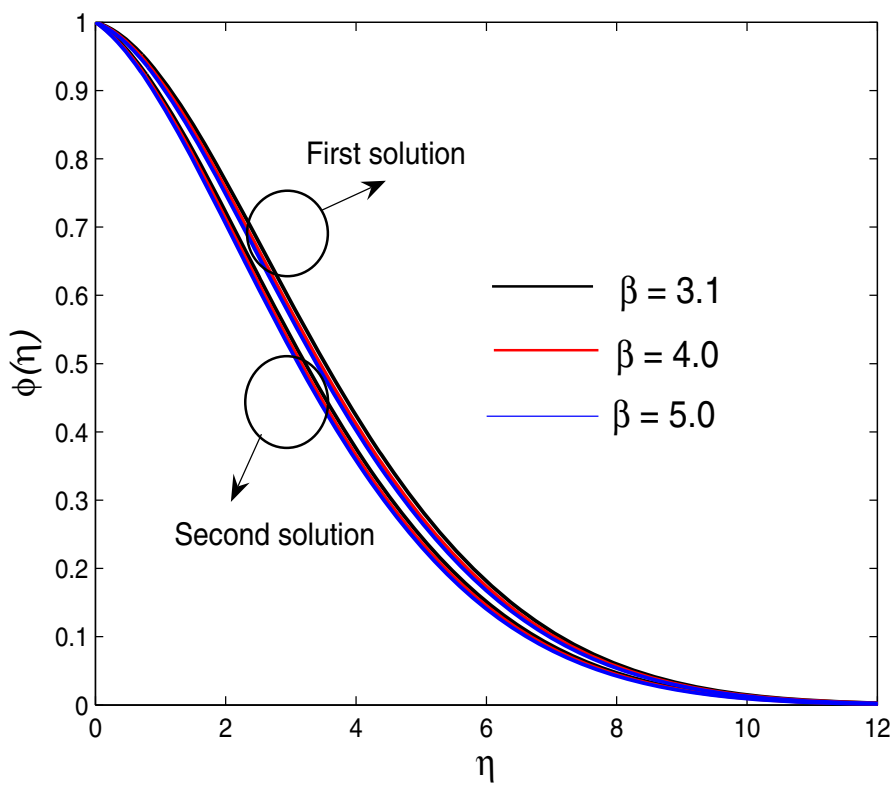

Figure 5. Effects of the Casson parameter on the concentration profiles when $\lambda=0.01, \operatorname{Pr}=D_{f}=$ $S c=S r=0.1, c / a=-1.25$.

case of a Casson fluid when compared with a viscous fluid. Hence in general, with an increase in $\beta$, the velocity of the fluid decreases for a stretching sheet. However, for a shrinking sheet the opposite is true and the velocity profiles increase with $\beta$. Consequently, the thermal and concentration boundary layer thicknesses decrease with an increase in $\beta$ in the case of a shrinking sheet.

\section{Conclusions}

The Soret and Dufour effects on a Casson fluid are investigated about a stagnation point on a stretching sheet. The momentum, temperature and concentration equations are written as a system of ordinary differential equations using a suitable similarity transformation and then solved numerically using the Matlab bvp4c package. Two solutions were obtained when $c / a<$ -1 . We have shown that Dufour number enhances the velocity and temperature throughout the boundary layer. The concentration boundary layer thickness is enhanced by an increase in the Soret number. The shrinking ratio reduces the velocity of the fluid but enhances the concentration layer thickness in the case of the first solution. The skin friction and heat and mass transfer rates increase with the Casson parameter.

\section{References}

Bhattacharyya K 2011 Dual solutions in boundarylayer stagnation-point flow and mass transfer with chemical reaction past a stretching/shrinking sheet. Int. Commun. Heat Mass Transf. 38: 917-922 
Bhattacharyya K, Hayat T and Alsaedi A 2013a Exact solution for boundarylayer flow of Casson fluid over a permeable stretching/shrinking sheet. ZAMM Z. Angew. Math. Mech. 1-7 doi: 10.1002/zamm.201200031

Bhattacharyya K, Hayat T and Alsaedi A 2013b Analytic solution for magnetohydrodynamic boundary layer flow of Casson fluid over a stretching/shrinking sheet with wall mass transfer. Chin. Phys. B. 22: 024702

Casson M 1959 A flow equation for pigment-oil suspensions of the printing ink type. In: C.C.Mills (ed.), Rheology of disperse systems, Newyork, Oxford: Pergamon, 84-104

Chamkha A J and Aly A M 2010 Heat and mass transfer in stagnation point flow of a polar fluid towards a stretching surface in porous media in the presence of Soret, Dufour and chemical reaction effects. Chem. Eng. Comm. 198: 214-234

Crane L J 1970 Flow past a stretching plate. J. Appl. Math. Phys. 21: 645-647

Dutta B K, Roy P and Gupta A S 1985 Temperature field in flow over a stretching sheet with uniform heat flux. Int. Commun. Heat Mass Transf. 12: 89-94

Hayat T, Javed T and Sajid M 2008 Analytic solution for MHD rotating flow of a second grade fluid over a shrinking surface. Phys. Lett. A 372: 3264-3273

Hayat T, Shehzad S A and Alsaedi A 2012a Soret and Dufour effectson magnetohydrodynamic (MHD) flow of Casson fluid. Appl. Math. Mech. Engl. Ed. 33: 1301-1312

Hayat T, Shehzad S A, Alsaedi A and Alhothuali M S 2012b Mixed convection stagnation point flow of casson fluid with convective boundary conditions. Chin. Phys. Lett. 29: 114704

Ishak A, Lok Y Y and Pop I 2010 Stagnation-point flow over a shrinking sheet in a micropolar fluid. Chem. Eng. Comm. 197: 1417-1427

Ishak A, Lok Y Y and Pop I 2012 Non-Newtonian power-law fluid flow past a shrinking sheet with suction. Chem. Eng. Commun. 199: 142-150

Kameswaran P K, Narayana M, Sibanda P and Murthy P V S N 2012 Hydromagnetic nanofluid flow due to a stretching or shrinking sheet with viscous dissipation and chemical reaction effects. Int. J. Heat Mass Transf. 55: 7587-7595

Kameswaran P K, Shaw S, Sibanda P and Murthy P V S N 2013 Homogeneous - heterogeneous reactions in a nanofluid flow due to a porous stretching sheet. Int. J. Heat Mass Transf. 57: 465-472

Liao S J 2003 On the analytic solution of magnetohydrodynamic flows of non-Newtonian fluids over a stretching sheet. J. Fluid Mech. 488: 189-212

Mahapatra T R and Gupta A S 2001 Magnetohydrodynamic stagnation-point flow towards a stretching sheet. Acta Mechanica. 152: 191-196

McDonald D A 1974 Blood flows in arteries. 2nd Edition, Chap. 2., London: Arnold

Merkin J H 1985 On dual solutions occurring in mixed convection in a porous media. J. Eng Math. 20: $171-179$

Merrill K, Beauchesne M, Previte J, Paullet J and Weidman P 2006 Final steady flow near a stagnation point on a vertical surface in a porous medium. Int. J. Heat Mass Transf. 49: 4681-4686

Mukhopadhyay S 2013 Casson fluid flow and heat transfer over a nonlinearly stretching surface. Chin. Phys. B 22: 7074701

Mustafa M, Hayat T, Pop I and Hendi A A 2012 Stagnation-point flow and heat transfer of a Casson fluid towards a stretching sheet. Z. Naturforsch 67: 70-76

Nakamura M and Sawada T 1988 Numerical study on the flow of a non-Newtonian fluid through an axisymmetric stenosis. J. Biomech Eng. 110: 137-143

Nawaz M, Hayat T and Alsaed A 2012 Dufour and Soret effects on MHD flow of viscous fluid between radially stretching sheets in porous medium. Appl. Math. Mech. 33: 1403-1418

Paullet J and Weidman P 2007 Analysis of stagnation point flow toward a stretching sheet. Int. J. NonLinear Mech. 42: 1084-1091

Postelnicu A and Pop I 2011 Falkner-Skan boundary layer flow of a power-law fluid past a stretching wedge. Appl. Math. Comput. 217: 4359-4368

Shaw S, Gorla R S R, Murthy P V S N and Ng C O 2009 Pulsatile Casson fluid flow through a stenosed bifurcated artery. Int. J. Fluid Mech. Res. 36: 43-63 
Shehzad S A, Hayat T, Qasim M and Asghar S 2013 Efects of mass transfer on MHD flow of Casson fluid with chemical reaction and suction. Brazilian J. Chem. Eng. 30: 187-195

Srinivasacharya D and RamReddy Ch 2011 Soret and Dufour effects on mixed convection in a non-Darcy porous medium saturated with micropolar fluid. Nonlinear Analysis: Modelling and Control 16: 100115

Wang C Y 2008 Stagnation flow towards a shrinking sheet. Int. J. Non-Linear Mech. 43: 377-382 